\title{
In-vivo imaging of microcirculation using integrated photoacoustic and optical-coherence microscopy
}

Li Li, Konstantin I. Maslov, Geng Ku, Lihong V. Wang

Li Li, Konstantin I. Maslov, Geng Ku, Lihong V. Wang, "In-vivo imaging of microcirculation using integrated photoacoustic and optical-coherence microscopy," Proc. SPIE 7177, Photons Plus Ultrasound: Imaging and Sensing 2009, 71770I (24 February 2009); doi: 10.1117/12.809323

SPIE. Event: SPIE BiOS, 2009, San Jose, California, United States 


\title{
In vivo imaging of microcirculation using integrated photoacoustic and optical coherence microscopy
}

\author{
Li Li*, Konstantin I. Maslov*, Geng Ku*, Lihong V. Wang*, \\ * Optical Imaging Laboratory, Washington University in St. Louis, St. Louis, Missouri, USA \\ 63130 \\ $\S$ Corresponding author. Email: lhwang@biomed.wustl.edu
}

\begin{abstract}
Photoacoustic imaging and optical coherence tomography have complementary imaging contrasts. Photoacoustic imaging is sensitive to optical absorption, thus is able to generate detailed maps of deep microvasculature in vivo. Optical coherence tomography exploits the optical scattering contrast, and can provide real-time, micrometer-resolution imaging of tissue. We integrate an optical-resolution photoacoustic microscopy and a spectral-domain optical coherence tomography into a single system. Our preliminary experiments showed that it could be a valuable imaging tool for microcirculation studies in vivo.
\end{abstract}

\section{Key words:}

photoacoustic, optical coherence tomography, microscopy, microcirculation, multi-modality imaging

\section{INTRODUCTION}

During the last century, the clinical practice and biomedical research have been revolutionized by the development of non-invasive imaging techniques, such as X-ray computed tomography (CT), positron emission tomography (PET), ultrasonography, magnetic resonance imaging (MRI) and optical imaging. However, an individual imaging modality usually cannot provide the comprehensive anatomical, functional and molecular information demanded by the physicians and investigators. For example, X-ray CT and ultrasonography are excellent tools for anatomical examinations even in real time, but they are not good at obtaining molecular and functional information due to the lack of molecular-specific contrast mechanisms. To the contrast, PET and optical tomography are good at visualizing functional and molecular events in vivo, nevertheless their images sometimes are difficult to interpret due to the lack of the concurrent structural content. Although MRI carries the promise to convey the three-level information by a single tool, but its sensitivity to physiological changes is found low in practice. Hence, recently multi-modality scheme is proposed to meet this challenge by incorporating two or more imaging techniques into a single system. The most successful example is PET/CT [1], which usually produces coregistered maps of metabolic activity (by PET) and anatomical structure (by CT). Nowadays, PET/CT has already become an indispensable tool for clinical cancer diagnostics. Another example is MRI-guided optical tomography or spectroscopy $[2,3]$, where functional parameters are assessed by optical methods using the MRI anatomical map as priori information. Its potential applications in brain imaging and breast cancer detection are currently under intensive investigation.

Photons Plus Ultrasound: Imaging and Sensing 2009, edited by Alexander A. Oraevsky, Lihong V. Wang, Proc. of SPIE Vol. 7177, 71770I · C 2009 SPIE · CCC code: 1605-7422/09/\$18 · doi: 10.1117/12.809323 
In this paper, we propose that the combination of photoacoustic imaging (PAI) and optical coherence tomography (OCT) could be the next paradigm of the multi-modality imaging. First, PAI and OCT have completely complementary imaging contrasts. PAI, although in its youth, has seen a rapid growth in the past few years [4]. PAI exploits the optical absorption contrast, which is directly associated with physiologically important molecules, such as hemoglobin. Thus, it not only can produce detailed map of deep vasculature in vivo, but also is capable of quantify the local total concentration of hemoglobin and oxygen saturation of blood in each single vessel utilizing the spectral information [5-7]. In the contrast, OCT [8], which is relatively mature, exploits the optical scattering contrast. State-of-art OCT can provide real-time, micrometer-resolution biopsy of tissue in vivo. Also, using the Doppler principle, depth-resolved profile of the blood flow can be estimated. Second, through proper selection of parts, the penetration depth and spatial resolution of the two modalities can match.

By combining the merits of the two techniques into a single system, we expect the resultant dual-modality system not only can image the detailed morphology of the microvasculature in relation with its surrounding structures, but also can quantify important functional parameters of each single vessel, like the total concentration of hemoglobin, oxygen saturation of blood and volumetric blood flow. Also, as demonstrated in previous works in PAI [9, 10], we can study molecular-specific events in vivo with the assistance of extraneous contrast agents. The potential application includes but is not limited to microcirculation study, ophthalmic diagnosis and tumor angiogenesis research.

\section{METHODS}

\subsection{System setup}

We have developed a first generation system following the above dual-modality strategy, called the integrated photoacoustic and optical coherence microscopy. It consists of three major parts: the imaging probe, the optical coherence subsystem and the photoacoustic laser. The imaging probe was configured similar to a traditional transmission microscope (Figure 1). The entire probe was loaded on an $\mathrm{x}$-y motorized stage. The light from the optical coherence subsystem and the photoacoustic laser were delivered to the probe through single-mode optical fibers. Compared with the free-space delivery scheme used in a previous system [7], the current design added flexibility to the device, and allowed scanning the probe instead of the sample. The light was then focused by a microscope objective (NA 0.25, PHACO1, Leitz Wetzlar, Germany) into the sample from the bottom. The sample was usually loaded on a standard glass slide. A high-frequency ultrasonic transducer $(75 \mathrm{MHz}, \mathrm{V} 2022 \mathrm{BC}$, Panametrics-NDT) was placed on top of sample to pick up the photoacoustic signal. An acoustic lens was attached to the transducer to achieve a focused geometry with a focal length of $5 \mathrm{~mm}$. During experiment, a drop of water was usually applied between the sample and the acoustic lens for better ultrasonic coupling. 


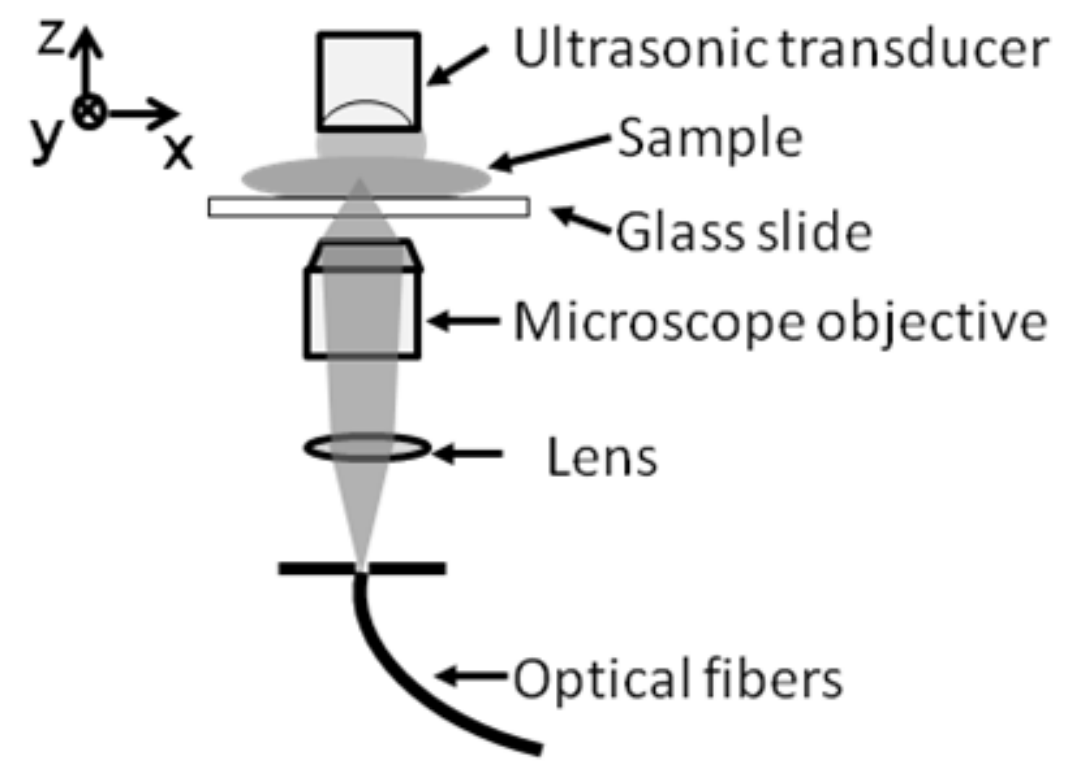

Figure 1 Configuration of the imaging probe.

The optical-coherence system adopted a standard spectral-domain configuration (Figure 2). Compared with its time-domain counterparts, this configuration has been proved to have higher sensitivity and faster imaging speed [11, 12]. It was based on a Michelson interferometer seeded by a superluminescent diode $\left(\lambda_{0}=829 \mathrm{~nm}, \Delta \lambda=36.4 \mathrm{~nm}\right.$, IPSDD0803, Inphenix). The axial resolution of the optical-coherence measurement was determined by the source bandwidth to be $8.4 \mu \mathrm{m}$ in air or $5.9 \mu \mathrm{m}$ in soft tissue (assume refractive index $\mathrm{n}=1.4$ ). The source light was split by a 50/50 fiber coupler into two arms. The object arm directly connected to the imaging probe, illuminated the sample and collected the backscattered light from it. The reference arm contained a retro-reflective mirror and was kept at a fixed length during experiment. The dispersion and polarization difference between the two arms were carefully compensated for. The light scattered back from the sample and the reference mirror was recombined by the same fiber coupler, and the interference signal was detected by a home-made spectrometer. The spectrometer was designed to have a spectral resolution of $67.5 \mathrm{pm}$, which limited the ranging depth of the optical-coherence system to be $2.5 \mathrm{~mm}$ in air or $1.8 \mathrm{~mm}$ in soft tissue. The system can acquire a maximum of 29,000 A-lines per second restricted only by the camera speed in the spectrometer. The optical coherence system was working in the reflection mode.

The photoacoustic subsystem worked in the transmission mode. It was powered by a short-pulse diode-pumped laser ( $\lambda=532 \mathrm{~nm}$, pulse width: $1.2 \mathrm{~ns}$, SPOT-10-100-532, Elforlight). The light out of the laser was coupled into a single-mode fiber which connected to the imaging probe. During experiment, the laser was fired at an average speed of $2 \mathrm{KHz}$, and pulse energy was estimated to be $\sim 10 \mathrm{~nJ}$ at the exit of the fiber. The transverse resolution of the photoacoustic image was determined by the optical focusing, and was quantified to be $\sim 1.5 \mu \mathrm{m}$. At the receiving only mode, this transducer was previously measured to have a FWHM bandwidth of $100 \mathrm{MHz}$ [7]. The axial 
resolution was mainly limited by ultrasonic bandwidth and the acoustic attenuation in sample, and was estimated to be $\sim 14 \mu \mathrm{m}$. Further details about the photoacoustic subsystem can be found in Ref [13].

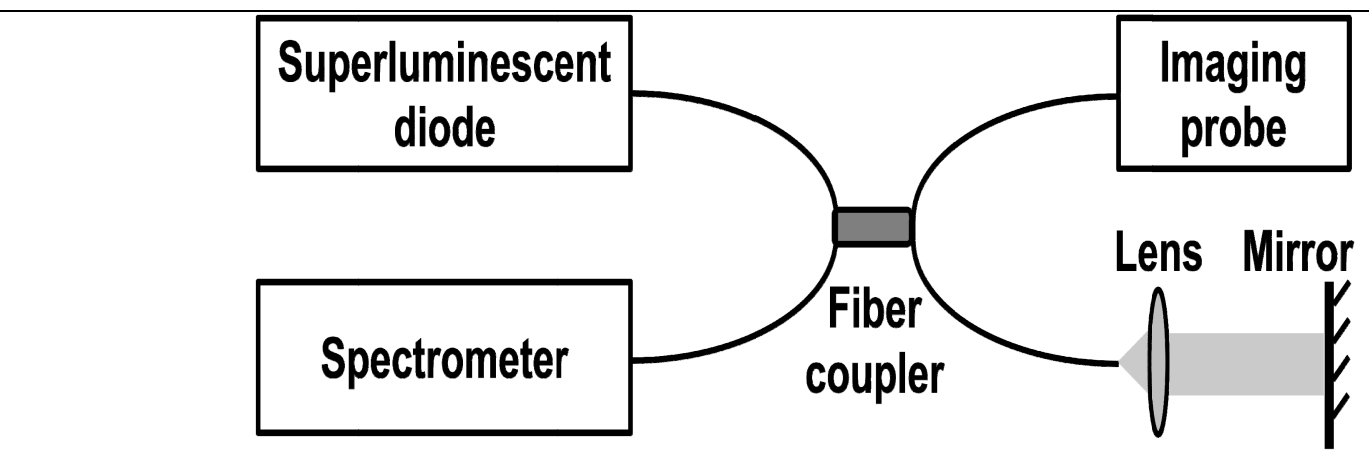

Figure 2 Schematic of the optical-coherence subsystem.

The data and control flow of the integrated dual-modality system were illustrated in Figure 3. The photoacoustic signal detected by the transducer was first amplified and then digitized by a NI-DAQ card. The optical-coherence spectrum was grabbed from the spectrometer using a NI-IMAQ card. The signal generated by the motor driver during scanning was also used to synchronize the firing of the photoacoustic laser and the data acquisition. A typical dual-contrast experiment generated two volumetric datasets, and usually covered a transverse area of $1 \mathrm{~mm} \times$ $1 \mathrm{~mm}$. This volume was typically covered by $800 \mathrm{~B}$-scans (x-z), and each B-scan included 800 A-lines (z). Each dataset with the above parameters was acquired within 12 minutes.

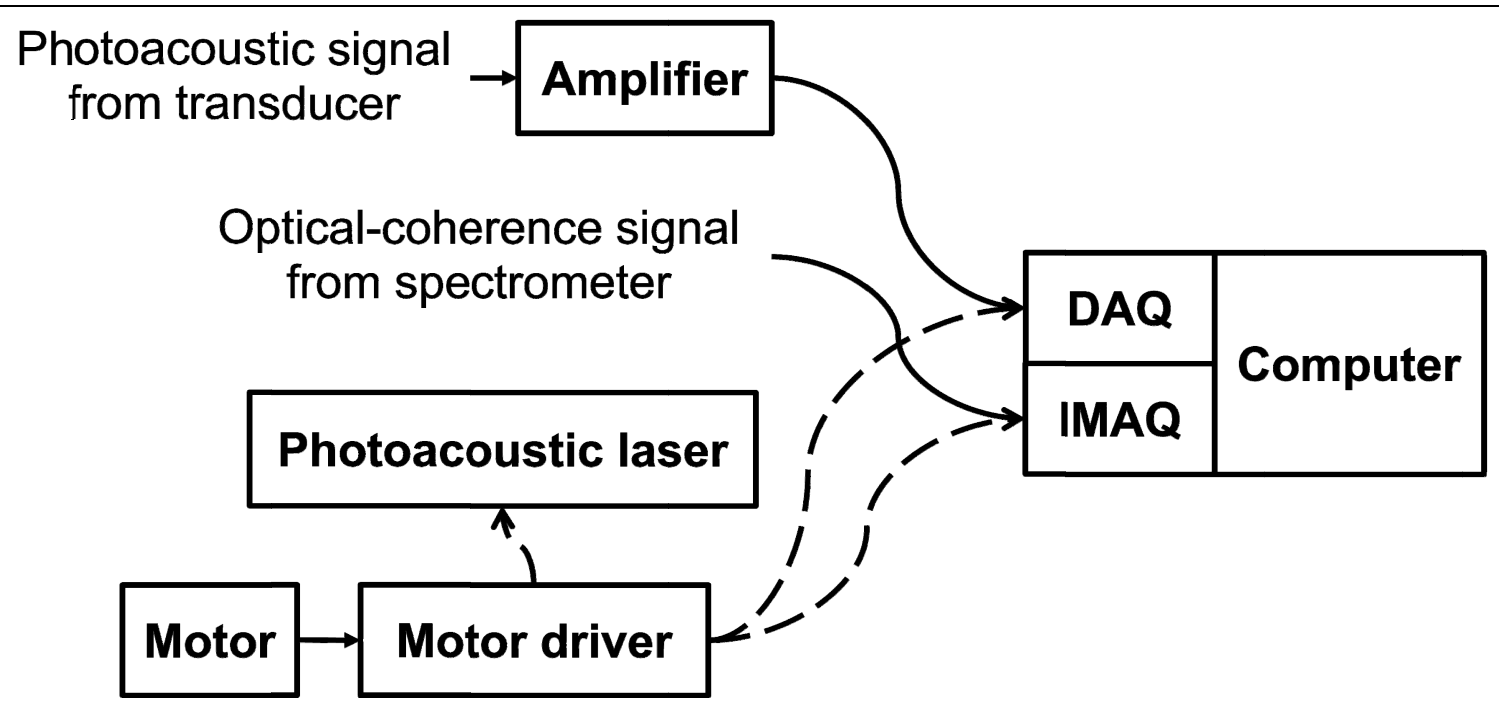

Figure 3 Schematic of the measurement and control signal flow of the dual modality system

\subsection{Animal model}


As an initial demonstration, we chose to image a standard animal model - the nude mouse ear. The nude mouse ear has been long recognized as an ideal in-vivo model for studying cutaneous microcirculation with optical methods.[14] The ear roughly measures $\sim 13 \times 13 \mathrm{~mm} 2$ with a thickness of $\sim 300 \mu \mathrm{m}$. It is composed of two fullthickness layers of skin separated by a thin supporting skeleton of elastic cartilage. The skin of the hairless mouse ear closely resembles human skin. Another attracting feature of this model is its special microcirculation pattern in the ear. The vasculature of a mouse ear originates from three pairs of arterioles and venules at the base of the ear. They normally bifurcate 4 times before the precapillary arterioles and postcapillary venules and capillary beds at the edge of the ear. As a result, this model allows us to investigate microcirculation at different levels by studying different area of the ear.

\section{RESULTS}

\subsection{Single-contrast images}

Figure 4 shows two single-contrast images of typical $x-z$ cross-sections of nude mouse ears obtained by the photoacoustic subsystem and the optical-coherence subsystem in vivo, respectively. The photoacoustic image (Figure 4a), representing absorbing strength, clearly shows the spatial distribution of micro-vessels. The smallest vessels were estimated to have a diameter of $7 \mu \mathrm{m}$, which were believed to be capillaries. However, the information of surrounding tissue was missing. To the contrary, the optical-coherence image (Figure $4 \mathrm{~b}$ ), which is sensitive to optical scattering, shows the microanatomy of ear. It resembles histology well (data not shown). We were also able to identify several obvious structures. The bright layer appearing both on the top and bottom of the ear was epidermis. The dark line in the middle was a layer of cartilage. The dark holes embedded in the skin layers were the glands. However, the micro-vessels are not apparent here due to the lack of sensitivity to optical absorption in optical coherence tomography. This is exactly the motivation why we want to combine the two modalities.

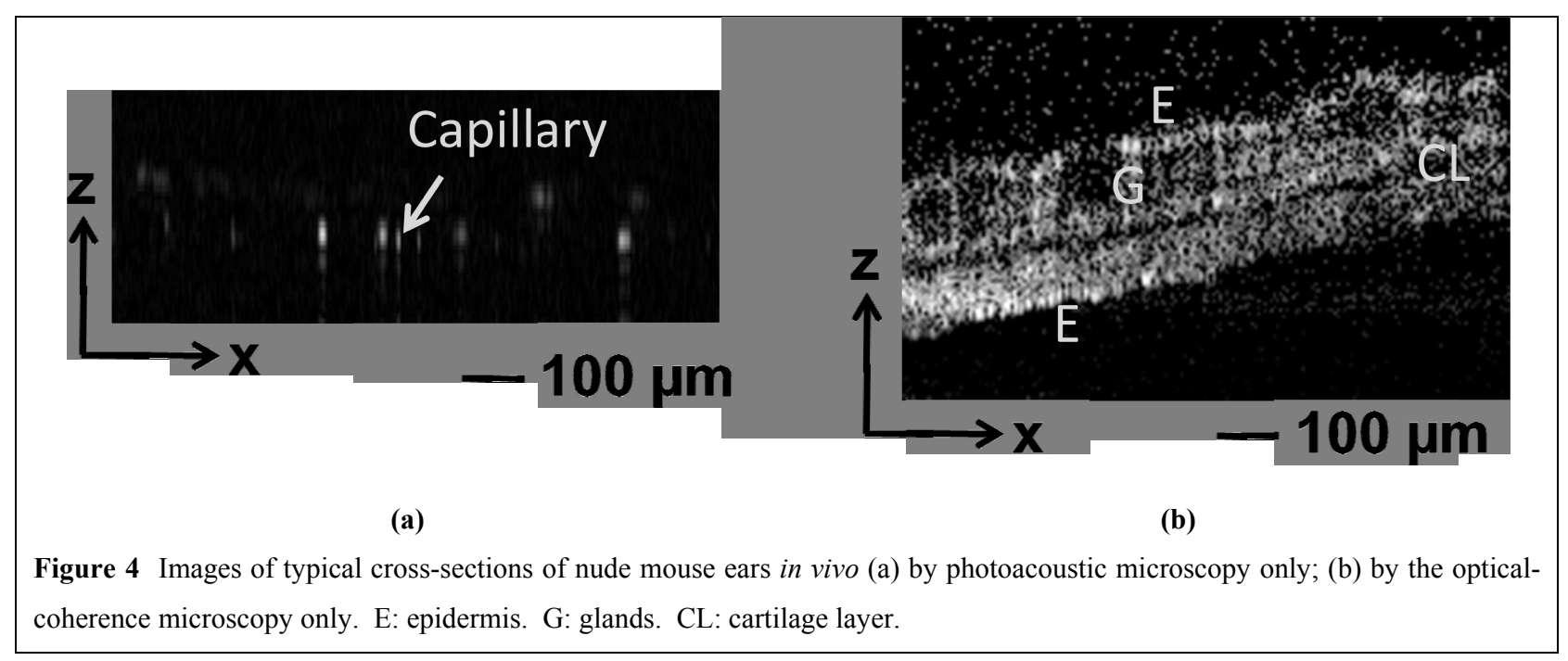




\subsection{Dual-contrast image}

Both photoacoustic and optical-coherence datasets were acquired over the same volume of a nude mouse ear during the same experiment in vivo. Composite images were then formed by superposing the blood vasculature mapped by photoacoustic subsystem (in red) over the anatomy imaged by the optical-coherence subsystem (in gray). A typical dual-contrast image of this kind is shown in Figure 5. The bright layer at the bottom represented the interface between the nude mouse ear and a glass slide. By exploiting both contrasts, we could identify the anatomical locations of vessels without ambiguity, and found they are actually distributed on both the top and bottom skin layers. What's more interesting, several capillaries were visualized and found to locate closer to the epidermis than other vessels. This matches the histological knowledge that the capillary loops in the mouse ear model are located in the sub-papillary layer and other vessels are located in the sub-dermal layer.

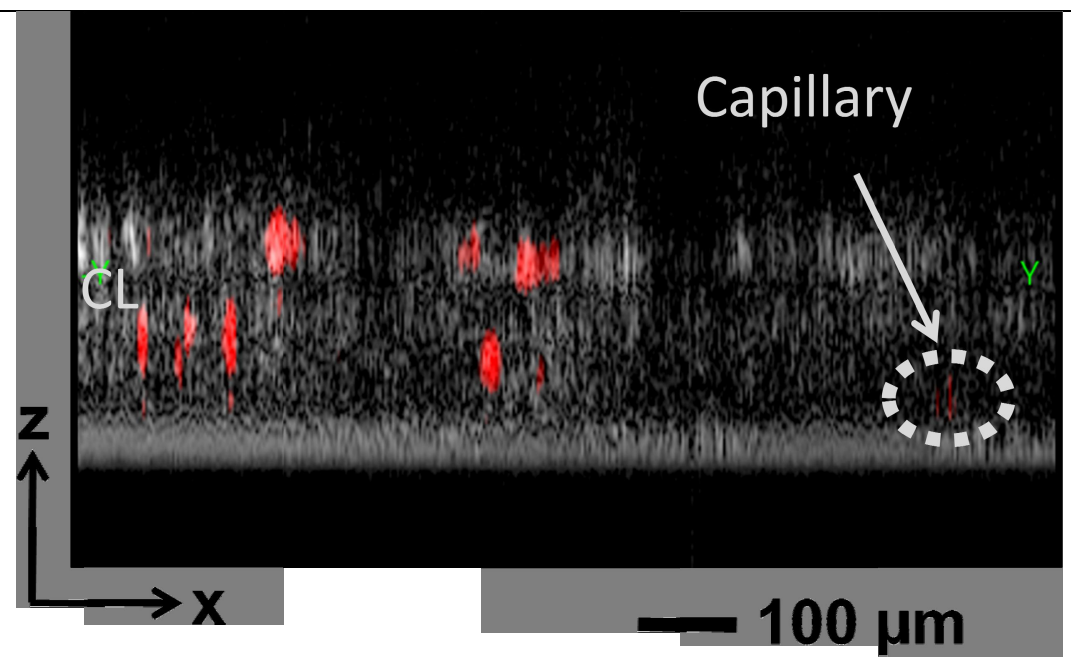

Figure 5 A typical dual-contrast cross-sectional image of a nude mouse ear by the integrated photoacoustic and optical coherence microscopy in vivo. This image is produced with composite colors (see the digit publication). The red features represent blood vessels, which were imaged by the photoacoustic subsystem. The gray features represent the surrounding tissue visualized by the optical-coherence subsystem. CL: cartilage layer.

\section{DISCUSSION}

In summary, we proposed a novel multi-modality imaging scheme, which combines the photoacoustic imaging and optical coherence tomography. We have developed the first generation system of this kind - the integrated photoacoustic and optical-coherence microscopy. The initial application, which we were targeted at, was to study the cutaneous microcirculation using nude mouse ear as a model. Our preliminary results demonstrated that the dual-modality scheme not only can map the morphology of microvasculature down to the capillary level using photoacoustic methods, but also can image the microanatomy of the surrounding soft tissue well using opticalcoherence techniques. Also, by co-registering the two, we were able to visualize the spatial relationship between the 
microcirculation and its surrounding tissue. Now, we are working to add the functional and molecular imaging capabilities to the system, and to extend the biological applications of the dual-modality tool.

\section{ACKNOWLEGEMENTS}

This work was sponsored in part by National Institutes of Health grants R01 CA092415, R01 NS46214 (BRP), R01 EB000712, R01 EB008085, and U54 CA136398 (NTR). L.W. has a financial interest in Endra, Inc., which, however, did not support this work.

\section{REFERENCES}

1. T. Beyer, D. W. Townsend, T. Brun, P. E. Kinahan, M. Charron, R. Roddy, J. Jerin, J. Young, L. Byars, and R. Nutt, "A combined PET/CT scanner for clinical oncology," J Nucl Med 41, 1369-1379 (2000).

2. V. Ntziachristos, A. G. Yodh, M. D. Schnall, and B. Chance, "MRI-guided diffuse optical spectroscopy of malignant and benign breast lesions," Neoplasia 4, 347-354 (2002).

3. X. F. Zhang, V. Y. Toronov, and A. G. Webb, "Integrated measurement system for simultaneous functional magnetic resonance imaging and diffuse optical tomography in human brain mapping," Rev Sci Instrum 77, 114301 (2006).

4. L. V. Wang, "Tutorial on Photoacoustic Microscopy and Computed Tomography," Selected Topics in Quantum Electronics, IEEE Journal of 14, 171-179 (2008).

5. X. D. Wang, Y. J. Pang, G. Ku, X. Y. Xie, G. Stoica, and L. V. Wang, "Noninvasive laser-induced photoacoustic tomography for structural and functional in vivo imaging of the brain," Nat Biotechnol 21, 803-806 (2003).

6. H. F. Zhang, K. Maslov, G. Stoica, and L. V. Wang, "Functional photoacoustic microscopy for highresolution and noninvasive in vivo imaging," Nat Biotechnol 24, 848-851 (2006).

7. K. Maslov, H. F. Zhang, S. Hu, and L. V. Wang, "Optical-resolution photoacoustic microscopy for in vivo imaging of single capillaries," Opt Lett 33, 929-931 (2008).

8. D. Huang, E. A. Swanson, C. P. Lin, J. S. Schuman, W. G. Stinson, W. Chang, M. R. Hee, T. Flotte, K. Gregory, C. A. Puliafito, and J. G. Fujimoto, "Optical Coherence Tomography," Science 254, 1178-1181 (1991).

9. L. Li, R. J. Zemp, G. Lungu, G. Stoica, and L. V. Wang, "Photoacoustic imaging of lacZ gene expression in vivo," Journal of Biomedical Optics 12, 020504 (2007).

10. M. L. Li, J. T. Oh, X. Y. Xie, G. Ku, W. Wang, C. Li, G. Lungu, G. Stoica, and L. V. Wang, "Simultaneous molecular and hypoxia imaging of brain tumors in vivo using spectroscopic photoacoustic tomography," Proceedings of the Ieee 96, 481-489 (2008). 
11. J. F. de Boer, B. Cense, B. H. Park, M. C. Pierce, G. J. Tearney, and B. E. Bouma, "Improved signal-tonoise ratio in spectral-domain compared with time-domain optical coherence tomography," Opt Lett 28, 2067-2069 (2003).

12. R. Leitgeb, C. K. Hitzenberger, and A. F. Fercher, "Performance of fourier domain vs. time domain optical coherence tomography," Opt Express 11, 889-894 (2003).

13. G. Ku, K. I. Maslov, L. Li, and L. V. Wang, "Photoacoustic microscopy with optically defined transverse resolution," Proceedings of SPIE 7177 (2009).

14. J. H. Barker, F. Hammersen, I. Bondar, E. Uhl, T. J. Galla, M. D. Menger, and K. Messmer, "The Hairless Mouse Ear for Invivo Studies of Skin Microcirculation," Plast Reconstr Surg 83, 948-959 (1989). 